\title{
FEM analysis of 3D lattice structures of ABS material
}

\author{
Seong-Gyu Cho ${ }^{1}$, Joon-Seong Lee ${ }^{2 *}$ \\ ${ }^{1}$ Dept. of Mechanical Engineering, Graduate School, Kyonggi University, 154-42, Gwanggyosan-ro, \\ Yeongtong-gu, Suwon-si, Gyeonggi-do, 16227, Korea. \\ ${ }^{2}$ Dept. of Mechanical System Engineering, Kyonggi University, 154-42, Gwanggyosan-ro, Yeongtong-gu, \\ Suwon-si, Gyeonggi-do, 16227, Korea. \\ *Corresponding author. Tel.: +82-10-3725-1140; Email address: jslee1@kyonggi.ac.kr
}

Article History:Received:11 november 2020; Accepted: 27 December 2020; Published online: 05 April 2021

\begin{abstract}
FDM is a typical additive manufacturing method. Since FDM is a method of stacking layers one by one, it generally has a flat lattice structure. In this study, by checking the distribution of stress and deformation for several lattice structures made of ABS material, it is intended to find a structure with better mechanical properties with less material. Several three-dimensional lattice structures are modeled using parametric modeling. Subsequently, a constant pressure is applied to the same area to check the stress and strain distribution. A structure with a low maximum stress value in the stress concentration region and a small amount of deformation will have the best mechanical properties. To do this, parametric modeling is performed using Inventor to model four three-dimensional lattice structures. Afterwards, use Ansys Workbench to check the stress and deformation distribution. Looking at the stress distribution, stress concentration occurred in the truss supporting the upper surface of the SC structure. In the BCC and PTC structures, stress concentration occurred at the point where the upper surface and the truss met. In the FCC structure, it can be seen that the load is distributed throughout the truss structure. Looking at the deformation distribution, both the SC and BCC structures show similar amounts of deformation. It was confirmed that the FCC structure had less maximum deformation than the PTC structure with the thickest truss. Unlike previous studies, it was confirmed that the higher the internal filling rate, the better the mechanical properties may not come out. The FDM method can obtain different mechanical properties depending on the internal lattice structure as well as the internal filling rate. In a later study, we will find a new calculation algorithm that applies variables by FDM characteristics using the data obtained by printing the actual specimen.
\end{abstract}

Keywords: ABS, Deformation, FEM analysis, Lattice, Stress distribution, Parametric modeling.

\section{Introduction}

Additive manufacturing is receiving a lot of attention in that it wastes less material than traditional subtractive manufacturing[1-3]. In addition, unlike casting or extrusion, the volume and weight can be properly adjusted by making the empty space inside the material free to some extent. And it is possible to print out parts of complex shape relatively easily. This feature makes it possible to reduce weight while having the appropriate strength and elasticity for the part of the desired shape. However, there is a disadvantage that the printing time is increased if the inside is filled and printed, such as cutting. In addition, since FDM is a method of outputting a plane layer by layer, it was common to fill the internal structure with a plane pattern. This is made into a structure that is more susceptible to a vertical load than to receive a horizontal load in the stacking direction. In order to solve this problem to some extent, if the internal filling structure is composed of a three-dimensional shape, it will be possible to secure similar strength and elasticity for loads in all directions.

To this end, the internal filling structure is composed of a certain lattice pattern, and the distribution of stress and strain for each pattern is checked through FEM simulation. In addition, when modeling the lattice pattern, the filling rate per unit volume can be easily changed by using parameters.

Ansys Workbench was used for FEM simulation, and Inventor was used for geometry modeling. In this study, the stress and deformation distribution of the three-dimensional lattice structures for the same parameter were checked to find a pattern excellent in stress and for the unit lattice.

\section{Materials and methods}

In the past, studies on the properties of FDM printouts of various materials have been conducted by several researchers[4-8]. Among them, the materials commonly used in FDM are PLA and ABS. These two materials have also been studied for various properties of FDM printouts[9-11]. Among them, ABS has superior mechanical properties compared to PLA and is often used as a mechanical part. Therefore, in this study, a simulation was performed by constructing a lattice with ABS material.

\subsection{Properties of ABS Material}

The properties of commonly used ABS materials are shown in Table 1 below.

Table1 General ABS properties using FEM Analysis

\begin{tabular}{|c|c|c|c|c|}
\hline Item & Density & $\begin{array}{l}\text { Young's } \\
\text { Modulus }\end{array}$ & Poison's Ratio & $\begin{array}{l}\text { Tensile Strength } \\
\text { [Yield] }\end{array}$ \\
\hline
\end{tabular}




\begin{tabular}{lllll}
\hline Value & $1.07 \mathrm{~g} / \mathrm{mm}^{\wedge} 3$ & $2.30 \mathrm{GPa}$ & 0.364 & $45 \mathrm{MPa}$ \\
& & & \\
\hline
\end{tabular}

\subsection{Shape of Lattice Structures}

As for the three-dimensional lattice structure, the Brave lattice is most commonly known. In this study, simple, body-centered, and face-centered cubic structures, which are Brave lattice structures, were selected, and additionally, a plate truss structure was considered. These structures are shown in Figure 1 below.

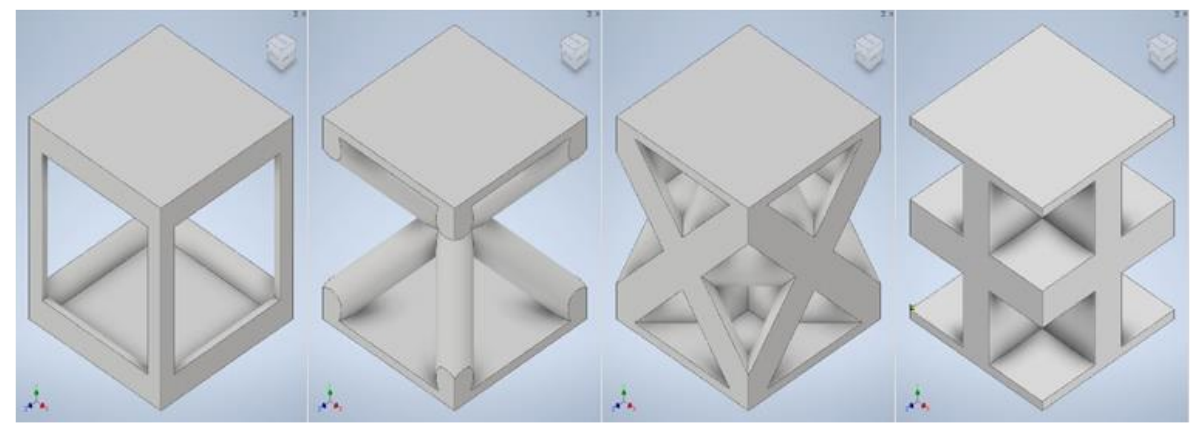

Figure 13 -D Lattice Structures using FEM Analysis

The lattice structure is a simple cubic lattice, a body-centered cubic lattice, a face-centered cubic lattice, and a plate truss lattice in order from the left. The position of the nodes of each lattice is the same as the atomic center of the Brave lattice structure. The truss connecting the nodes is the same as the shortest distance between atoms.

\subsection{Parametric Modeling}

The lattice structure has the shape of a cube and is composed of a truss of a certain thickness. Therefore, the parameters applied to the modeling consisted of the length 10 of the lattice and the thickness (or diameter) d0 of the truss. The applied parameter values are shown in Table 2 below.

Table2 Parametric values applied to the lattice structure

\begin{tabular}{lll}
\hline Item & DO & LO \\
\hline Value & $4 \mathrm{~mm}$ & $20 \mathrm{~mm}$ \\
\hline
\end{tabular}

In FDM, the minimum output width is determined according to the size of the nozzle. In this study, the nozzle diameter was assumed to be $0.1 \mathrm{~mm}$ and the minimum output width was set at $0.2 \mathrm{~mm}$. Therefore, the value of the parameter was determined as an integer multiple of $0.2 \mathrm{~mm}$.

Table 3 shows the internal filling rates of each lattice to which the parameter values of Table 2 are applied.

Table3 Comparison of Internal Filling Rate of each Lattice Structures

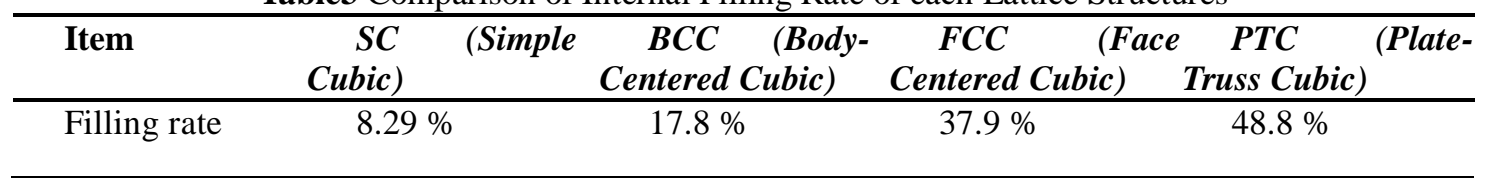

\subsection{Applied Boundary Conditions}

Gravity was applied to all lattice structures, and the part that touched the ground was fixed. Also, a pressure of $0.1 \mathrm{MPa}$ was applied to the top surface. In order to deliver pressure evenly over the same area, a $1 \mathrm{~mm}$ thick plate was added to the top and bottom of each lattice structure. Boundary conditions applied to FE analysis are shown in Table 4.

Table4 Boundary Conditions Applied to FEM Analysis

\begin{tabular}{lll}
\hline Item & Value & Note \\
\hline Load (Pressure) & $0.1 \mathrm{MPa}$ & Top surface / -y direction
\end{tabular}




\begin{tabular}{lll} 
Fixed Support & All DOF & Bottom surface \\
Gravity (Self-weight) & $9.81 \mathrm{~m} / \mathrm{s}^{\wedge} 2$ & -y direction \\
\hline
\end{tabular}

\section{Results}

The FEM analysis results are shown in Figure 2 to 5 below. Each analysis result is a simple cubic lattice, body-centered cubic lattice, face-centered cubic lattice, and plate truss lattice in order from the left.

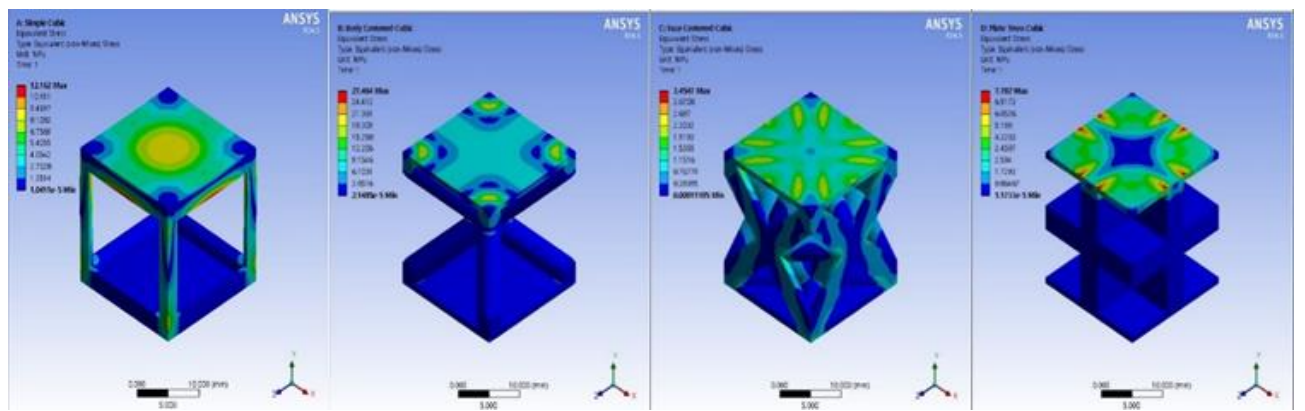

Figure 2 Stress Distribution of each Lattice Structures

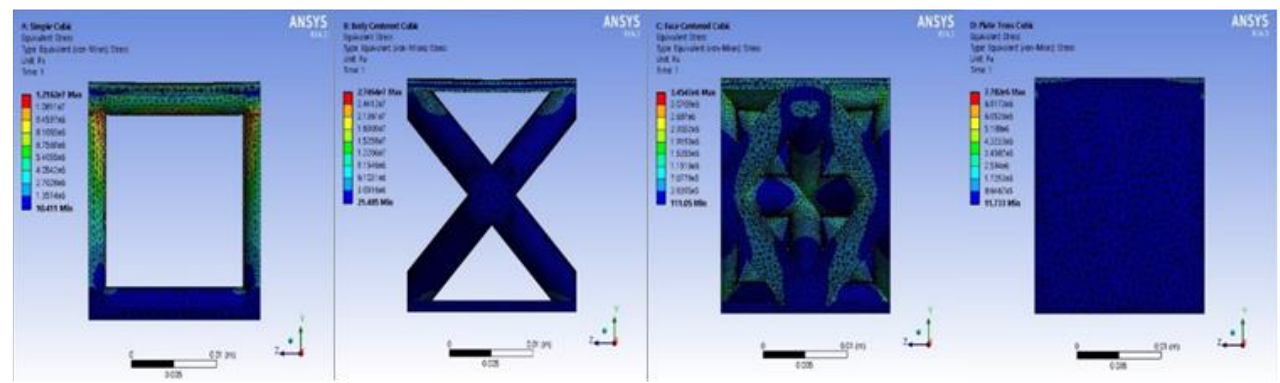

Figure 3 Stress Distribution of each Lattice Structures (Mid-plane)

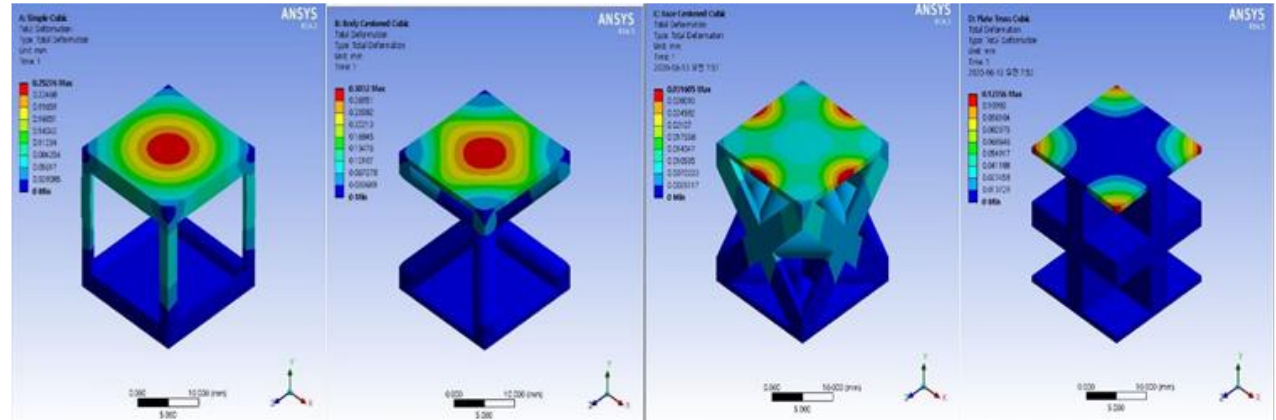

Figure 4 Deformation Distribution of each Lattice Structures

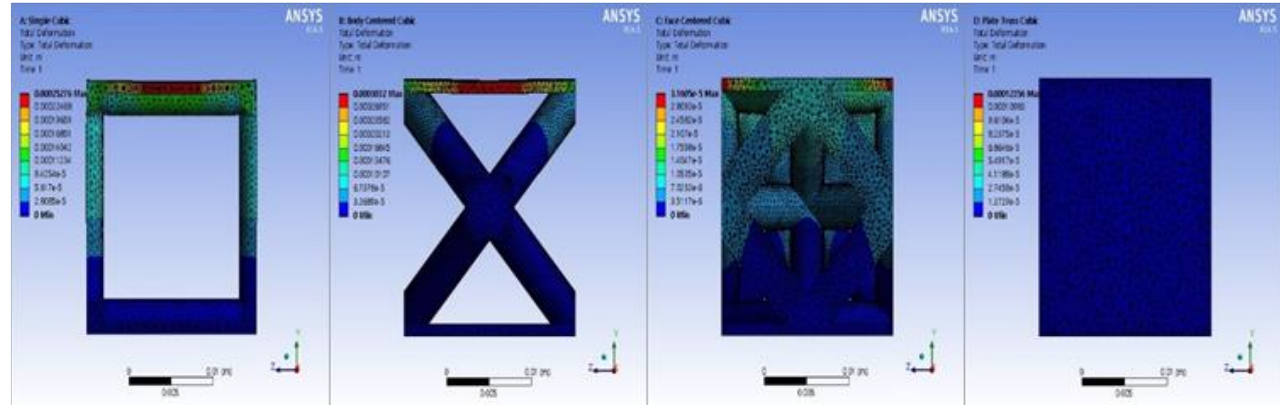

Figure 5 Deformation Distribution of each Lattice Structures (Mid-plane)

Table 5 and Table 6 summarize the analysis results in a simple table.

Table5 Maximum Stress of Lattice Structures 


\begin{tabular}{|c|c|c|c|c|}
\hline Item & $S C$ & $B C C$ & $F C C$ & PTC \\
\hline Stress & $12.161 \mathrm{MPa}$ & $27.464 \mathrm{MPa}$ & $3.4547 \mathrm{MPa}$ & $7.787 \mathrm{MPa}$ \\
\hline \multicolumn{5}{|c|}{ Table6 Maximum Deformation of Lattice Structures } \\
\hline Item & $S C$ & $B C C$ & $F C C$ & PTC \\
\hline Deformation & $0.25276 \mathrm{~mm}$ & $0.3032 \mathrm{~mm}$ & $0.031605 \mathrm{~mm}$ & $0.12356 \mathrm{~mm}$ \\
\hline
\end{tabular}

\section{Discussion}

Looking at the stress distribution, in the SC structure, a lot of stress concentration occurs in the truss supporting the upper surface. In the BCC structure, stress concentration occurs at the point where the truss and the thin plate meet. In the FCC structure, it is confirmed that the stress is evenly distributed throughout the structure. In the case of the PTC structure, the truss is thick, so the stress is not evenly distributed in the truss itself, and stress concentration occurs in the area that is joined to the top surface. It can be seen that the structure in which the highest stress concentration occurs is the BCC structure, and the structure that evenly distributes the stress overall is the FCC structure.

Looking at the deformation distribution, it shows the highest amount of deformation at the center of the plane that the truss cannot support in all structures. Both SC and BCC showed high deformation, and the PTC with the thickest truss showed about four times higher deformation than FCC. It is believed that this is because the FCC structure truss distributes the stress evenly compared to the PTC structure truss.

\section{Conclusions}

In this study, the stress distribution and deformation distribution for 3 Brave lattice and 1 plate- truss lattice were confirmed. The structure with the least maximum stress is the FCC structure, and the structure with the least maximum deformation is the FCC structure. The structure with the second least maximum stress is the PTC structure, and the structure with the second least maximum deformation is the PTC structure. In the case of the Brave lattice, the maximum stress and maximum deformation were high even though the BCC structure had more than twice the internal filling rate of the SC structure. In addition, although the PTC structure had a higher internal filling rate than the FCC structure, the stress and deformation were higher.

According to previous FDM studies, the higher the internal filling rate, the less the amount of deformation was. However, in this study, it was confirmed that the mechanical properties may differ depending on the shape of the lattice structure along with the internal filling rate.

A brief summary of the conclusions obtained in this study is as follows.

1) The higher the internal filling rate, the less stress and deformation unconditionally appear.

2) FDM output may exhibit different mechanical properties depending on the internal lattice structure as well as the internal filling rate.

This study did not consider the change in the physical properties of the FDM output for the air gap, and did not consider the difference between the general ABS properties and the ABS properties during the FDM output. It should be recalled that this is not an accurate result because it does not take into account many of the variables that apply to the FDM output. In addition, it should be recalled that the FEM analysis algorithm did not reflect the FDM characteristics because the general stress calculation formula was used.

In the future studies, we will print out actual specimens, measure using UTM, and conduct a linkage study on more precise analysis algorithms for FDM outputs through FEM analysis applying a new calculation formula considering several variables.

\section{Acknowledgements}

This work was supported by GRRC program of Gyeonggi province. [GRRC 2020-0200, Research on Innovative Intelligent Manufacturing System]

\section{References}

1. Drummer D, Cuellar SC, Rietzel D. Suitability of PLA/TCP for fused deposition modeling.

2. Rapid Prototyping Journal. 2012 Sep;18(6): 344-357. DOI:10.1108/13552541211272045

3. Xinhua L, Shengpeng L, Zhou L, Xianhua Z, Xiaohu C, Zhonbin W. An investigation on distortion of PLA thin-plate part in the FDM process. Int J Adv Technol. 2015 Feb;79: 1117-1126. DOI:10.1007s00170-015-6893-9 
4. Song Y, Li Y, Song W, Yee K, Lee KY, Tagarielli VL. Measurements of the mechanical response of unidirectional 3D-printed PLA. Materials\&Design. $2017 \quad$ Jun;123: 154-164. DOI:10.1016/j.matdes.2017.03.051

5. Kang YG, Kweon HK, Shin GS. Strength Variation with Inter-Layer Fill Factor of FDM 3D Printer. Journal of the Korean Society of Manufacturing Process Engineers. 2019 Mar;18(3):66-73. DOI:10.14775/ksmpe.2019.18.3.066

6. Kim BJ, Lee HT, Sohn IS. A study of mechanical properties with FDM 3D printing layer conditions. Journal of the Korea Society of Die \& Mold Engineering. 2018 Dec;12(3):19-24.

7. Ziemian CW, Cipoletti DE, Ziemian SN, Okwara MN, Haile KV. Monotonic and Cyclic Tensile Properties of ABS Components Fabricated by Additive Manufacturing. Annual International Solid Freeform Fabrication Symposium 2014:525-41

8. Seol KS, Shin BC, Zhang SU. Fatigue Test of 3D-printed ABS Parts Fabricated by Fused Deposition Modeling. Journal of the Korean Society of Manufacturing Process Engineers. 2018 Jun;17(3):93-101. DOI:10.14775/ksmpe.2018.17.3.093

9. Jin JW, Jeong BG, Park UW, Kang KW. Evaluation of Buckling Failure Behavior of Cylindrical Lattice Structures Manufactured by 3D Printer. Trans Korean Soc Mech Eng A. 2020 Aug;44(8):58995. DOI:10.3795/KSME-A.2020.44.8.589

10. Choi W, Woo JH, Jeon JB, Yoon SS. Measurement of Structural Properties of PLA Filament as a Supplier of 3D Printer. Journal of The Korean Society of Agricultural Engineers. 2015 Nov;57(6):14152. DOI:10.5389/KSAE.2015.57.6.141

11. Yoon J. Study of the mechanical properties and orthotropy of ABS materials fabricated by FDM printing. Journal of the Korean Society of Manufacturing Process Engineers. 2018 Oct;17(5):143-8. DOI:10.14775/ksmpe.2018.17.5.143

12. Afrose MF, Masood SH, Iovenitti P, Nikzad M, Sbarski I. Effects of part build orientations on fatigue behaviour of FDM-processed PLA material. Progress in Additive Manufacturing. 2015 Nov;1:21-8. 